\title{
The hunt for new pulsars with the Green Bank Telescope
}

\author{
Ryan S. Lynch ${ }^{1}$, \\ on behalf of the GBT $350 \mathrm{MHz}$ Drift-scan survey ${ }^{2}$ \\ and Green Bank North Celestial Cap survey ${ }^{3}$ collaborations $^{2}$ \\ ${ }^{1}$ Department of Physics, McGill University, \\ 3600 University Street, Montreal, QC H3A 2T8, Canada \\ email: rlynch@physics.mcgill.ca \\ ${ }^{2}$ http://www . as. wvu.edu/ pulsar/GBTdrift350/ \\ ${ }^{3}$ http://arcc.phys.utb.edu/gbncc/
}

\begin{abstract}
The Green Bank Telescope (GBT) is the largest fully steerable radio telescope in the world and is one of our greatest tools for discovering and studying radio pulsars. Over the last decade, the GBT has successfully found over 100 new pulsars through large-area surveys. Here I discuss the two most recent- the GBT $350 \mathrm{MHz}$ Drift-scan survey and the Green Bank North Celestial Cap survey. The primary science goal of both surveys is to find interesting individual pulsars, including young pulsars, rotating radio transients, exotic binary systems, and especially bright millisecond pulsars (MSPs) suitable for inclusion in Pulsar Timing Arrays, which are trying to directly detect gravitational waves. These two surveys have combined to discover 85 pulsars to date, among which are 14 MSPs and many unique and fascinating systems. I present highlights from these surveys and discuss future plans. I also discuss recent results from targeted GBT pulsar searches of globular clusters and Fermi sources.
\end{abstract}

Keywords. Pulsars: general, surveys

\section{Introduction}

Since their discovery 45 years ago, just over 2000 pulsars have been uncovered, enabling some of the most precise and fascinating astronomical discoveries over that same time span. The majority of these are radio pulsars that have been found in large-area surveys, though a significant fraction, especially of millisecond pulsars (MSPs), were found in targeted searches. Almost all surveys result in some new and often unexpected discovery, many of which have an impact beyond the study of neutron stars. Some highlights from only the past few years are: the double pulsar (Lyne et al.2004), which has provided some of the best tests of strong-field general relativity (Kramer et al. 2006), as well pulsar-white dwarf systems that place stringent limits on tensor-vector-scalar theories of gravity (Bhat et al. 2008; Lazaridis et al. 2009; Freire et al. 2012); PSR J1614-2230, a $2 \mathrm{M}_{\odot}$ MSP that has provided the best constraints yet on the equation of state of ultradense matter (Demorest et al.2010); the unexpected discovery of a population of gammaray pulsars and MSPs (Abdo et al.2009a; Abdo et al.2009b); the discovery of rotating radio transients (RRATs) and the implication that they may outnumber traditional radio pulsars (McLaughlin et al. 2006); and three radio-emitting magnetars (Camilo et al. 2006; Camilo et al.2007; Levin et al.2010) that have shed light on the relationship between radio pulsars and magnetars. One of the most exciting prospects for the future is the direct detection of gravitational waves using a pulsar timing array (PTA) of ultra-high precision MSPs, which will help to usher in a new era of gravitational wave astronomy. 
New pulsar surveys are important for continuing this tradition of discovery, especially in light of the need for more high-precision MSPs for use in PTAs. Different surveys often complement each other owing to different sky coverage and observing set-ups (e.g. high frequency surveys are more sensitive to distant pulsars with high dispersion measures (DMs), while low-frequency surveys are more sensitive to steep-spectrum pulsars). The National Radio Astronomy Observatory $\dagger$ (NRAO) in Green Bank, West Virginia has a long history of discovering fascinating pulsars through large-area, low radio frequency surveysł. The first of these low-frequency Green Bank pulsar surveys was carried out using the 92-m telescope by Damashek et al. (1978) at an observing frequency of $400 \mathrm{MHz}$ and uncovered 17 pulsars. This was followed up by Dewey et al. (1985) and Stokes et al. (1985), also using the $92-\mathrm{m}$ telescope at $390 \mathrm{MHz}$. The latter survey was sensitive to pulsars with periods $4 \mathrm{~ms} \leqslant P \leqslant 100 \mathrm{~ms}$ but did not find any, leading the authors to conclude that the number of such pulsars must be small compared to long-period pulsars and providing early evidence that the then-newly discovered MSPs constituted a separate population with a distinct evolutionary history. Sayer et al. (1997) discovered a MSP (defined here as having $P<20 \mathrm{~ms}$ ) and a $P=40.9 \mathrm{~ms}$ relativistic binary using the $43-\mathrm{m}$ telescope at $370 \mathrm{MHz}$.

The Robert C. Byrd Green Bank Telescope (GBT) was built to replace the 92-m telescope and is the largest fully steerable telescope in the world. Over the past decade, the GBT has proven to be one of the best (and perhaps even the best) pulsar telescopes in the world, having discovered over 200 pulsars, many of which are MSPs. This is due to its excellent sensitivity and suite of low-noise receivers, its location in an environment with relatively little radio frequency interference (RFI), and state-of-the art pulsar back-ends. The first low-frequency large-area survey carried out with the GBT was the $350 \mathrm{MHz}$ North Galactic Plane survey (Hessels et al. 2008), which discovered 33 pulsars. The GBT has also revolutionized the field of globular cluster (GC) pulsars, uncovering 71 GC MSPs, more than any other telescope. It has also been instrumental in identifying radio counterparts to Fermi-selected pulsars and MSPs. Here, I will discuss the two most recent large-area GBT surveys, the GBT $350 \mathrm{MHz}$ Drift-scan survey and the Green Bank North Celestial Cap (GBNCC) survey. I will also briefly discuss the GBT's role in targeted searches of GCs and Fermi sources.

\section{The $350 \mathrm{MHz}$ Drift-scan Survey}

The Drift-scan survey was completed between 2007 May and August while the GBT was closed for normal operations due to repair of the azimuth track. The main science motivation for the survey was the discovery of high-precision MSPs suitable for inclusion in the North American Nanohertz Observatory for Gravitational Waves $₫$ (NANOGrav), as well as other exotic pulsars. It covered a variety of right ascensions between declinations $-7.7^{\circ} \leqslant \delta \leqslant 38.4^{\circ}$ and $-20.7^{\circ} \leqslant \delta \leqslant 38.4^{\circ}$ (see Figure 2) using $50 \mathrm{MHz}$ of bandwidth and 140-s long sections of data. In total, $10347 \mathrm{deg}^{2}$ were observed\|, totaling some 134 TB. Further details of the survey coverage, data processing, and sensitivity can be found in Boyles et al. (2012) and Lynch et al. (2012). Data processing for the Driftscan survey is now complete, and 35 pulsars have been discovered, including 7 MSPs

$\dagger$ NRAO is a facility of the National Science Foundation operated under cooperative agreement by Associated Universities, Inc.

$\ddagger$ I am grateful to Jason Boyles for compiling this list.

I http://nanograv.org

\| $2800 \mathrm{deg}^{2}$ were reserved for the Pulsar Search Collaboratory (Rosen et al.2010). See http://www.pulsarsearchcollaboratory.org/ 


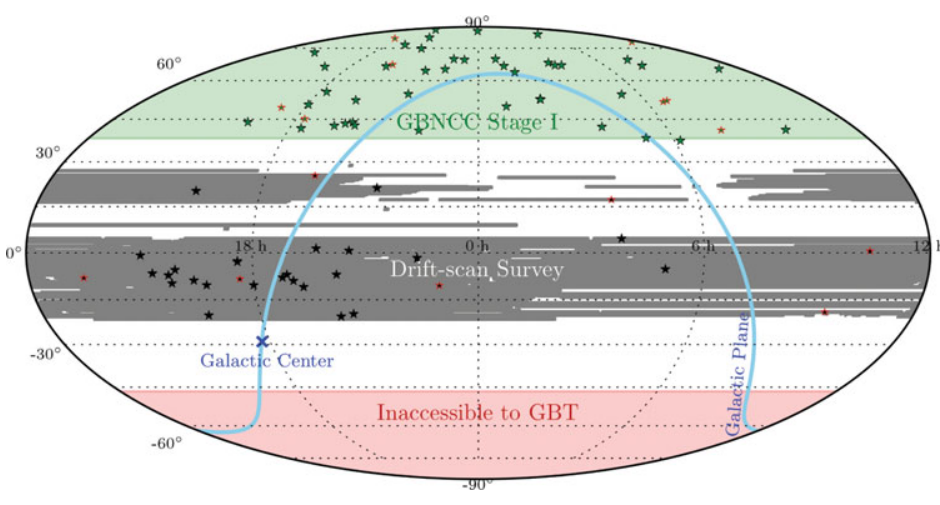

Figure 1. Sky coverage of the Drift-scan and GBNCC Stage I surveys. New pulsars are shown as stars, and MSPs are outlined in red. The GBNCC survey is currently being extended to lower declinations with the goal of eventually covering the entire sky visible to the GBT.

and recycled pulsars. Twenty-four pulsars from early data processing are presented in Archibald et al. (2009), Boyles et al. (2012), and Lynch et al. (2012) along with complete timing solutions. An additional 11 pulsars have been discovered since this first round of detailed follow-up and are still being studied. Below I provide a few highlights.

- PSR J0348+0432 is a 39-ms recycled pulsar in a short, 2.4-hr orbit around a white dwarf companion (Lynch et al.2012). Optical imaging and spectroscopic followup of the companion have provided tight constraints on the mass of the white dwarf (Antoniadis et al., in prep), which is about $0.17 \mathrm{M}_{\odot}$. The different binding energies of the pulsar and white dwarf are predicted by certain tensor-vector-scalar gravitational theories to lead to strong dipolar gravitational wave emission. Timing using the Arecibo telescope is already placing stringent limits on these theories in an as-yet unexplored strong-field regime (Antoniadis et al., in prep) and future timing will improve these constraints.

- PSR J0337+1715 is a 2.7-ms MSP in a hierarchical triple, the first to be discovered in the field of the Galaxy. The inner companion appears to be a white dwarf with an outer companion of undetermined nature on a much longer orbit orbit. This system was only recently discovered during final processing of the Drift-scan data, but early timing is already showing evidence for secular changes to the orbital parameters of the inner binary due to three-body interactions (Ransom et al., in prep), making this system a precision dynamical laboratory.

- PSR J2222-0137 is a 33-ms recycled pulsar with a minimum companion mass of $1.1 \mathrm{M}_{\odot}$ (Boyles et al.2012). The pulsar is nearby $(D=310 \mathrm{pc})$ and bright $\left(S_{820 \mathrm{MHz}} \sim\right.$ $2 \mathrm{mJy}$ ), and has been studied using the Very Long Baseline Array (Deller et al., in prep). The pulsar has also been the subject of a campaign to measure Shapiro delay (Boyles et al., in prep). These results will be presented in future publications.

- Two high-precision MSPs discovered in the Drift-scan survey have been released to NANOGrav and the International Pulsar Timing array.

- The well-known "missing link" MSP, PSR J1023+0038 (Archibald et al. 2009) was one of the earliest Drift-scan discoveries and has shed light on the connection between low-mass X-ray binaries and MSPs.

- Thirty-three RRAT candidates have been discovered in the Drift-scan, with about six having already been confirmed. This marks a substantial increase in the size of the RRAT population (for details see the discussion by Karako-Argaman, these proceedings). 


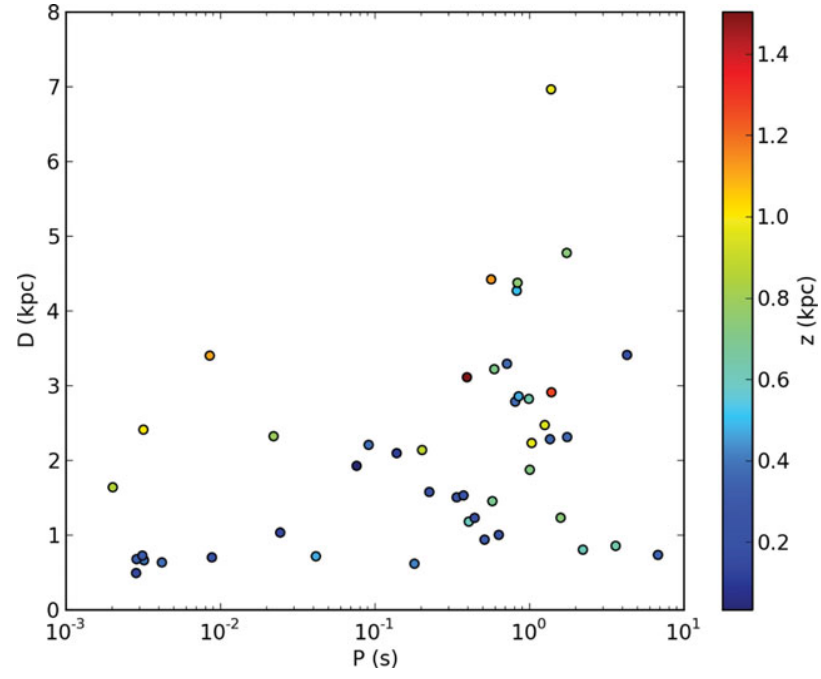

Figure 2. Spin periods and DM-inferred distances of newly discovered GBNCC pulsars. The color bar indicates the distance above the Galactic plane. Nearly a dozen MSPs have DM-inferred distances $\lesssim 1 \mathrm{kpc}$, making them promising candidates for astrometric and multiwavelength study.

\section{The Green Bank North Celestial Cap Survey}

The GBNCC survey is the successor to the Drift-scan survey and was also carried out at $350 \mathrm{MHz}$, giving it excellent sensitivity to nearby, steep spectrum pulsars. It uses twice the bandwidth of the Drift-scan survey (100 MHz), 120-s pointed integrations, and the newer Green Bank Ultimate Pulsar Processor back-end (DuPlain et al. 2008), which provides increased dynamic range, bandwidth, and better resistance to RFI (though there is already little RFI). The science goals are the same, but with a particular emphasis on northern declinations, where there are fewer high-precisions MSPs known, especially in the first stage of the survey. This is important for increasing the number of wideseparation baselines in PTAs and also probes a region of the Galaxy that has not been studied in as much detail as the Galactic plane. Stage I of the survey covered the north celestial cap $\left(\delta>38^{\circ}\right)$ and data taking was completed in 2011. We are currently conducting the second stage of the survey by moving towards lower declinations with the goal of eventually covering the entire sky visible to the GBT.

Data processing is being carried out at the Texas Advanced Computing Center $\dagger$ and especially using the Guillimin supercomputer operated by CLUMEQ $\ddagger$. These substantial computing resources (up to 2048 CPUs are reserved for pulsar searching on Guillimin) have allowed us to reduce most of the data collected thus far, though we have only managed to do a preliminary analysis of the resulting pulsar candidates. These early results, however, are quite promising, with 50 new pulsars $₫$, including 9 new MSPs\|. Detailed follow-up of these new pulsars is ongoing, but I provide some early highlights below.

- MSPs: Three of the nine new MSPs appear to be excellent candidates for PTAs, and indeed, the 8.85-ms MSP PSR J0645+51 has already been released to NANOGrav A. At least five MSPs are in binary systems. PSR J1816+4510 has a $>0.16 \mathrm{M}_{\odot}$

$\dagger$ http://www.tacc.utexas.edu/

$\ddagger$ http://www.clumeq.ca/index.php/en/about/computers/guillimin

ฯ Of the 50 new pulsars, 21 were identified by high school students through a summer internship program at McGill University, and 11 were identified by undergraduate students as part of the Advanced Arecibo Control Center program at the University of Texas at Brownsville and the University of Wisconsin-Milwaukee, demonstrating how education and outreach programs can be blended with real-world research.

\| See http://arcc.phys.utb.edu/gbncc/ for an up-to-date list. 
companion and has been detected with Fermi. There is also a UV/optical counterpart (Kaplan et al.2012). PSR J0636+51 is a 2.9-ms MSP in a 1.5-hr orbit. The lower limit on the companion mass is a factor of two higher than that of the so-called "diamond planet" (Bailes et al. 2011). PSR J1124+78 is a nearby $(D \sim 600 \mathrm{pc}$ ) binary MSP with a likely white dwarf companion, and we have proposed for time to look for an optical counterpart.

- Intermediate Period Pulsars: Five pulsars have periods $20 \mathrm{~ms} \leqslant P \leqslant 100 \mathrm{~ms}$, and four have Galactic latitudes $|b|>10^{\circ}$. These are properties often seen in doubleneutron star systems, and there is a possibility that at least one of these intermediate period pulsars is in a relativistic binary. The fifth has $b \approx 1^{\circ}$, and its proximity to the plane raises the possibility that it is a young pulsar, though no supernova remnants have been found at the position of the pulsar in radio and X-ray catalogs. Further investigation is needed to determine the nature of these pulsars.

- Nearby Pulsars: Eleven pulsars (including five MSPs) have DM-inferred distances $<1 \mathrm{kpc}$ (see Figure 3) according to the NE2001 free electron density model (Cordes \& Lazio2002), and several of these have high $350 \mathrm{MHz}$ flux densities. These are excellent candidates for obtaining parallax and proper motion measurements using very-long baseline interferometry, as well as for searching for counterparts at other wavelengths. One source in particular, PSR J0737+69, has a period of $6.8 \mathrm{~s}$, making it the fifth slowest rotating radio pulsar known (though there are several magnetars and $\mathrm{X}$-ray dim isolated neutron stars with similar or longer periods $\dagger$ ). It's proximity makes it an excellent candidate for X-ray follow-up.

A more detailed analysis of pulsar candidates from the GBNCC survey, as well as ongoing data-taking and processing, will undoubtedly result in the discovery of many more pulsars in the near future.

\section{Targeted Pulsar Searches with the GBT}

The GBT has proven itself to be a superb instrument for finding pulsars through targeted searches, notably of GCs and unidentified Fermi sources. Since 2004, nearly all new GC pulsars have been found with the GBT. In fact, the GBT has discovered 71 GC pulsars, nearly half of the entire known population $\ddagger$. The most promising GCs visible from the GBT have all been deeply searched (Ransom et al. 2004; Ransom et al. 2005; Freire et al. 2008; Lynch \& Ransom2011a; Lynch et al.2011b; Lynch et al. 2012; Stairs et al., in prep), with highlights including 34 pulsars in Terzan 5 (Ransom et al. 2005), the fastest known rotator (Hessels et al. 2006) and potential super-massive neutron stars (Freire et al. 2008). Though the pace of discovery has slowed in recent years, GC pulsars are still being discovered with the GBT. For a more detailed discussion of GC pulsars, see the discussion by Paulo Freire in these proceedings.

Fermi has revolutionized the search for field radio pulsars, particularly MSPs. Radio emission from 45 MSPs and four long-period pulsars have been discovered by targeting bright, unidentified Fermi sources, and 26 of these have been found using the GBT (see Ray et al. (2012) for a complete list of Fermi MSPs and their discovery telescopes). See the discussions by Pablo Saz Parkinson and Lucas Guillemot in these proceedings for a more detailed discussion of Fermi pulsar searches.

$\dagger$ See the ATNF pulsar database (http://www.atnf.csiro.au/people/pulsar/psrcat/) and the McGill SGR/AXP Online Catalog (http://www.physics.mcgill.ca/ pulsar/magnetar/ main.html).

$\ddagger$ See http://www.naic.edu/ pfreire/GCpsr.html for an up-to-date list. 


\section{Conclusions}

Exciting and unique pulsars are still being discovered almost anywhere they are looked for, and the GBT continues to play an indispensable role in this. The Drift-scan and GBNCC surveys have combined to discover 85 pulsars, including 16 MSPs or recycled pulsars, in addition to 33 strong RRAT candidates. These include some truly fascinating sources that are enabling cutting-edge physics and astronomy. The continuation of GBNCC survey ensures that more discoveries are yet to come. Searches of GC are still sensitivity limited, and searches of unidentified Fermi sources continue with the promise of yet more MSPs. As such, the GBT should continue in its role as one of the best pulsar telescopes in the world.

\section{References}

Abdo, A. A., Ackermann, M., Ajello, M., et al. 2009a, Science, 325, 840

Abdo, A. A., Ackermann, M., Ajello, M., et al. 2009b, Science, 325, 848

Antoniadis, J., Freire, P. C. C., Wex, N., et al. in prep

Archibald, A. M., Stairs, I. H., Ransom, S. M., et al. 2009, Science, 324, 1411

Bailes, M., Bates, S. D., Bhalerao, V., et al. 2011, Science, 333, 1717

Bhat, N. D. R., Bailes, M., \& Verbiest, J. P. W. 2008, Phys. Rev. D, 77, 124017

Boyles, J., Lynch, R., Ransom, S. M., et al. 2012, ApJ submitted, arXiv:1209.4293

Boyles, J., et al. in prep

Camilo, F., Ransom, S. M., Halpern, J. P., et al. 2006, Nature, 442, 892

Camilo, F., Ransom, S. M., Halpern, J. P., \& Reynolds, J. 2007, ApJ, 666, L93

Cordes, J. M. \& Lazio, T. J. W. 2002, arXiv:astro-ph/0207156

Damashek, M., Taylor, J. H., \& Hulse, R. A. 1978, ApJ, 225, L31

Deller, A. T., et al. in prep

Demorest, P. B., Pennucci, T., Ransom, S. M., Roberts, M. S. E., \& Hessels, J. W. T. 2010, Nature, 467, 1081

Dewey, R. J., Taylor, J. H., Weisberg, J. M., \& Stokes, G. H. 1985, ApJ, 294, L25

DuPlain, R., Ransom, S., Demorest, P., et al. 2008, Proc. SPIE, 7019, 45

Freire, P. C. C., Ransom, S. M., Bégin, S., et al. 2008, ApJ, 675, 670

Freire, P. C. C., Wolszczan, A., van den Berg, M., \& Hessels, J. W. T. 2008, ApJ, 679, 1433

Freire, P. C. C., Wex, N., Esposito-Farèse, G., et al. 2012, MNRAS, 423, 3328

Hessels, J. W. T., Ransom, S. M., Kaspi, V. M., et al. 2008, 40 Years of Pulsars: Millisecond Pulsars, Magnetars and More, 983, 613

Hessels, J. W. T., Ransom, S. M., Stairs, I. H., et al. 2006, Science, 311, 1901

Kaplan, D. L., Stovall, K., Ransom, S. M., et al. 2012, ApJ, 753, 174

Kramer, M., Stairs, I. H., Manchester, R. N., et al. 2006, Science, 314, 97

Lazaridis, K., Wex, N., Jessner, A., et al. 2009, MNRAS, 400, 805

Levin, L., Bailes, M., Bates, S., et al. 2010, ApJ, 721, L33

Lynch, R. S., Boyles, J., Ransom, S. M., et al. 2012, ApJ submitted, arXiv:1209.4296

Lynch, R. S., Freire, P. C. C., Ransom, S. M., \& Jacoby, B. A. 2012, ApJ, 745, 109

Lynch, R. S. \& Ransom, S. M. 2011a, ApJ, 730, L11

Lynch, R. S., Ransom, S. M., Freire, P. C. C., \& Stairs, I. H. 2011ba, ApJ, 734, 89

Lyne, A. G., Burgay, M., Kramer, M., et al. 2004, Science, 303, 1153

McLaughlin, M. A., Lyne, A. G., Lorimer, D. R., et al. 2006, Nature, 439, 817

Ransom, S. M., Hessels, J. W. T., Stairs, I. H., et al. 2005, Science, 307, 892

Ransom, S. M., Stairs, I. H., Backer, D. C., et al. 2004, ApJ, 604, 328

Ransom, S. M., Stairs, I. H., et al. in prep

Ray, P. S., Abdo, A. A., Parent, D., et al. 2012, arXiv:1205.3089

Rosen, R., Heatherly, S., McLaughlin, M. A., et al. 2010, Astronomy Education Review, 9, 010106

Sayer, R. W., Nice, D. J., \& Taylor, J. H. 1997, ApJ, 474, 426

Stairs, I. H., et al. in prep

Stokes, G. H., Taylor, J. H., Weisberg, J. M., \& Dewey, R. J. 1985, Nature, 317, 787 